\title{
Conséquences métaboliques des malabsorptions lipidiques : apports de I'étude des hypocholestérolémies familiales
}

\author{
Noël PERETTI \\ Hospices Civils de Lyon, \\ Hôpital Femme Mère Enfant de Lyon, \\ Bron, \\ F-69677, \\ France; \\ Université de Lyon, \\ INSERM U1060, \\ CarMeN laboratory, \\ Lyon, \\ F-69008 France; \\ Université Lyon 1, \\ Faculté de Médecine Lyon-Est, \\ Lyon, \\ F-69003, \\ France \\ <noel.peretti@chu-lyon.fr >
}

Article reçu le 11 mai 2012

Accepté le 13 juin 2012

\section{Introduction}

L'absorption intestinale des lipides joue un rôle majeur non seulement dans le contrôle des apports énergétiques, mais aussi au plan structural et métabolique. En effet, l'apport calorique lié à la fraction lipidique de notre alimentation est important, environ $95 \mathrm{~g} / \mathrm{j}$ dans I'alimentation française (Hercberg et al., 1998). Les récentes recommandations françaises ont même proposé une augmentation des apports nutritionnels conseillés en lipides jusqu'à 35-40 \% des apports caloriques journaliers (ANSES, 2011). De plus, les lipides sont des constituants essentiels pour la structure des membranes cellulaires ainsi que pour le système nerveux central. Enfin, les lipides sont les précurseurs de nombreuses molécules jouant un rôle fondamental au niveau

\begin{abstract}
Metabolic consequences of intestinal malabsorption of lipids: how studying hereditary hypocholesterolemia improves our knowledge

Familial hypocholesterolemia, namely abetalipoproteinemia, hypobetalipoproteinemia and chylomicron retention disease (CRD), are rare genetic diseases that cause intestinal lipid malabsorption. They provide a model to study the consequences of chronic hypocholesterolemia: protection against cardiovascular disease, increase of fatty liver disease and neurovascular complications. The understanding of their physiopathology provided new approaches to treat hypercholesterolemia.
\end{abstract}

Key words: familial hypocholesterolemia, abetalipoproteinemia, hypobetalipoproteinemia, chylomicron retention disease (CRD), intestinal lipid malabsorption, chronic hypocholesterolemia

métabolique : les hormones stéroïdiennes, la vitamine $D$ et les sels biliaires pour lecholestérol, ou bien certaines molécules del'inflammation commelesleucotriènes ou les prostaglandines métabolisées à partir de certains acides gras essentiels. Les conséquences d'une malabsorption lipidique risquent donc d'être multiples. Nous focaliserons cet exposé sur les conséquences des malabsorptions intestinales congénitales des lipides induisant une hypocholestérolémie.

Le cholestérol plasmatique est à $50 \%$ $d^{\prime}$ origine endogène par synthèse cellulaire principalement hépatique sous le contrôle notamment de l'enzyme HMG CoA reductase, et à $50 \%$ d'origine intestinale provenant non seulement de I'alimentation (300 à 500 mg/jour), mais aussi des acides biliaires (800 à $1200 \mathrm{mg} / \mathrm{j}$ ) ainsi que de la desquamation des cellules intestinales (250 à $400 \mathrm{mg} / \mathrm{j})$. Le cholestérol est absorbé de manière variable selon les individus avec une moyenne de $50 \%$ des apports (Sehayek et al., 1998), les extrêmes pouvant aller de $20 \%$ à $80 \%$ (Bosner et al., 1999).

Les causes de malabsorptions intestinales lipidiques sont nombreuses. On distingue classiquement d'une part les causes dites secondaires, c'est-à-dire qu'elles sont la conséquence d'une pathologie affectant la digestion comme les insuffisances pancréatiques ou certaines maladies hépatiques, ou bien altérant l'absorption intestinale elle-même soit par réduction de la surface d'absorption comme dans le syndrome de grêle court, soit par altération de l'épithélium intestinale comme dans les maladies inflammatoires chroniques de l'intestin par exemple. D'autre part, les causes primitives parmi lesquelles se trouvent les hypocholestérolémies génétiques comprenant actuellement trois pathologies distinctes: I'abétalipoprotéinémie (AB), I'hypobétalipoprotéinémie (HB) et la rétention de chylomicron (RCM) ou maladie d'Anderson. Elles représentent un groupe de maladies rares qui ont permis de progresser dans la compréhension de la physiologie de l'absorption intestinale lipidique. Elles peuvent être une aide conceptuelle à l'élaboration de nouvelles stratégies thérapeutiques pour les hypercholestérolémies dont l'impact en santé publique est majeur : en mimant I'anomalie physiopathologique causale, on espère obtenir

Pour citer cet article : Peretti N. Conséquences métaboliques des malabsorptions lipidiques : apports de l'étude des hypocholestérolémies familiales. OCL 2012 ; 19(4) : 228-231. doi : 10.1684/ocl.2012.0463 
une réduction de l'absorption intestinale du cholestérol et ainsi une baisse de I'hypercholestérolémie. Pour cette raison, il apparaît essentiel de s'interroger sur les conséquences métaboliques qui sont associées à une baisse de la cholestérolémie secondaire à une malabsorption intestinale pour le suivi des patients mais aussi pour comprendre les effets secondaires potentiels des nouvelles stratégies hypocholestérolémiantes élaborées à partir de ces maladies rares.

La définition de I'hypocholestérolémie pose plusieurs problèmes pratiques. En effet, la valeur moyenne du cholestérol plasmatique varie en fonction de l'origine ethnique, ainsi les Américains d'origine africaine présentent des taux moyens plus bas que ceux d'origine européenne (Bosner et al., 1999). La cholestérolémie varie également selon le sexe et l'âge : les hommes ayant des taux plus bas que les femmes entre 25 et 50 ans puis cette tendance s'inversant après la cinquantaine (NIH, 1980). Chez l'enfant, les taux varient selon l'âge également (Ford et al., 2009 ; Lambert et al., 1984). Enfin, la cholestérolémie est influencée non seulement par l'efficacité de l'absorption intestinale qui est variable selon les individus (Bosner et al., 1999), mais également la diète notamment sa composition en acides gras saturés. Cependant, en pratique, il est généralement admis comme seuil un taux de cholestérol total inférieur à 2,6 mmol/L $(1 \mathrm{~g} / \mathrm{L})$ et de LDL cholestérol inférieur à $1,56 \mathrm{mmol} / \mathrm{L}$ $(0,6 \mathrm{~g} / \mathrm{L})$.

\section{Les hypocholestérolémies d'origine intestinale}

L'absorption intestinale du cholestérol est sous la dépendance de la synthèse des chylomicrons par l'entérocyte. Trois étapes clés sont indispensables pour obtenir une sécrétion efficace des chylomicrons au pôle basolatéral de l'entérocyte après la digestion d'un repas. Premièrement, la synthèse de I'apolipoprotéine B au niveau du réticulum endoplasmique afin de produire I'ApoB48 qui assure la cohésion structurale du chylomicron ainsi que sa liaison au récepteur hépatique permettant ainsi la capture de la lipoprotéine. Une mutation du gène de I'ApoB est responsable de I'HB conduisant dans la moitié des cas à une protéine tronquée dont la fonctionnalité est altérée (Leppert et al., 1988 ; Wu et al., 1999). Deuxièmement, le transfert de lipides sur I'ApoB en cours de synthèse est indispensable pour prévenir la dégradation de cette protéine au niveau du protéasome. La Microsomal Triglycerid Transfert Protein (MTP) est un complexe protéique indispensable à cette étape de lipidation. La mutation du gène de la MTP se retrouve dans I'AB (Wetterau et al., 1992), elle est responsable d'une destruction précoce de I'ApoB par le protéasome empêchant ainsi la synthèse des chylomicrons. Enfin, troisièmement, le transfert du pré-chylomicron depuis le réticulum endoplasmique vers l'appareil de Golgi est nécessaire pour permettre la réalisation de la dernière étape de maturation avant la sécrétion du chylomicron. Ce transfert intra-entérocytaire est sous la dépendance de la protéine Sar1 GTPase. La mutation de son gène SAR1B est retrouvée dans la maladie de rétention des chylomicrons également appelée maladie d'Anderson (Jones et al., 2003).

Ces trois maladies ont pour conséquence une surcharge lipidique de l'entérocyte induisant une malabsorption sévère des lipides et une hypocholestérolémie chronique. Soulignons toutefois que les taux lipidiques sont plus sévèrement diminués dans I'hypobétalipoprotéinémie homozygote et l'abétalipoprotéinémie que dans la maladie de rétention des chylomicrons.

\section{Conséquences cardiovasculaires}

Le lien entre une valeur augmentée du LDL-C et le risque cardiovasculaire est parfaitement établi. De plus, I'abaissement de ces taux pathologiques réduit le risque cardiovasculaire. II semble donc raisonnable de poser l'hypothèse qu'un taux anormalement abaissé de cholestérolémie de manière chronique puisse prévenir la survenue d'un risque cardiovasculaire élevé. Les hypocholestérolémies familiales pouvant alors être envisagées comme un "modèle " de traitement intensif des hypercholestérolémies.

Le suivi à long terme de sujets porteurs d'hypobétalipoprotéinémie révèle une incidence faible d'accidents cardiovasculaires: une trentaine d'individus présentant un cholestérol LDL inférieur au $5^{\mathrm{e}}$ percentile ont bénéficiés d'un génotypage, la moitié d'entre eux présentaient une mutation du gène de I'ApoB. Ces individus étaient indemnes de toute maladie cardiovasculaire, toutefois la population est relativement jeune avec un âge moyen de 40 ans et peu d'information sur les risques cardiovasculaires sont fournis dans la publication (Fouchier et al., 2005). Cependant, dans une autre étude, il est intéressant de noter que même si l'épaisseur intima média (EIM) de patients porteur d'HB n'est pas différente de celle des sujets contrôles, leur rigidité vasculaire par contre est significativement diminuée malgré des facteurs de risques associés plus nombreux suggérant ainsi un effet protecteur de I'hypocholestérolémie chronique sur la fonctionnalité de la paroi vasculaire (Sankatsing et al., 2005).

II peut également exister une hypocholestérolémie chronique par d'autres mécanismes que la malabsorption intestinale. Ainsi, une augmentation de la captation hépatique du cholestérol circulant est décrite dans le cadre de polymorphismes de la protéine PCSK9 (Cohen et al., 2005). L'étude ARIC prospective sur une quinzaine $d^{\prime}$ années a permis de démontrer une diminution drastique du risque cardiovasculaire chez les sujets hypocholestérolémiques par variants PCSK9 : ainsi une diminution de $88 \%$ du risque est décrite avec le variant $\mathrm{C679X}$, et une diminution de $47 \%$ pour le variant R46L (Cohen et al., 2006).

En conclusion, les conséquences métaboliques au plan cardiovasculaire d'une hypocholestérolémie chronique par malabsorption intestinale des lipides semblent bien bénéfiques. Cependant la comparaison entre les hypocholestérolémies familiales et le traitement intensif des hypercholestérolémies présente plusieurs limites : tout d'abord, les effets propres des traitements qui viennent s'ajouter à l'effet direct de la baisse de la cholestérolémie comme par exemple l'effet pleïotrope des statines; d'autre part, les conséquences néfastes qui peuvent être associées aux hypocholestérolémies comme les cardiomyopathies décrites dans I'AB et la RCM (Silvain et al., 2008). Enfin, les carences en antioxydants importantes présentes lors des malabsorptions intestinales lipidiques pourraient également avoir des conséquences délétères au niveau cardiovasculaire. 
Parmi les innovations thérapeutiques inspirées de ces maladies rares, nous pouvons citer les oligonucléotides antisens anti-ApoB mimant la physiopathologie de I'HB hétérozygote et permettant une baisse du LDL cholestérol entre $40 \%$ et $88 \%$ dans un modèle murin hypercholestérolémique (Crooke et al., 2005).

\section{Conséquences hépatiques}

L'existence d'une hypocholestérolémie génétique expose au risque métabolique de stéatose hépatique et potentiellement de NASH. Les sujets avec hypocholestérolémie familiale ont souvent des taux de transaminases augmentés indiquant l'existence d'une cytolyse, les valeurs se situent habituellement dans une fourchette entre deux et trois fois les valeurs normales (Schonfeld et al., 2003). Bien que la physiopathologie exacte de cette cytolyse reste à préciser, il est établi que les sécrétions des triglycérides et d'ApoB100 par le foie sont diminuées (Elias et al., 1999) et que les hypocholestérolémies sont associées à une augmentation de la surcharge lipidique du foie; ainsi la quantification par IRM révèle jusqu'à 5 fois plus de lipides intrahépatocytaires chez les patients porteurs d'HB (Schonfeld et al., 2003). Les analyses histologiques montrent des lésions superposables à celle trouvée dans les états de NAFLD, les sujets porteurs d'HB sont à risque de développer des lésions histologiques plus sévères que des sujets contrôles présentant une stéatose hépatique d'une autre origine (Sankatsing et al., 2005). L'évolution vers la cirrhose a été rapportée pour I'AB et I'HB mais non pour la RCM jusqu'à présent (Avigan et al., 1984 ; Illingworth et al., 1980 ; Partin et al., 1974 ; Peretti et al., 2010). Soulignons, que les patients avec HB hétérozygotes, bien qu'asymptomatiques pourraient être quand même particulièrement susceptibles de développer des cirrhoses, ce qui peut faire discuter un suivi au long cours malgré l'absence de symptômes chez ces sujets (Tarugi et Averna, 2011).

Il est intéressant de rappeler ici que I'AB, en faisant découvrir le rôle essentiel de la MTP dans le contrôle de la sécrétion post-prandiale des chylomicrons et du taux de cholestérolémie, a permis de développer des inhibiteurs spécifiques de cette protéine dans le but de traiter les hypercholestérolémies. Parmi les effets secondaires de la première génération de ces inhibiteurs, une stéatose hépatique importante était un obstacle majeur au développement de ces nouveaux traitements malgré une efficacité remarquable chez I'humain avec une baisse de $70 \%$ du LDL-C (Chandler et al., 2003). Pour cette raison, des inhibiteurs de la MTP spécifiques de l'entérocyte ont pu être développés permettant d'obtenir l'effet hypocholestérolémiant souhaité, mais sans stéatose hépatique associée (Kim et al., 2011).

\section{Conséquences surrénaliennes}

Le cholestérol étant le substrat des hormones stéroïdiennes, il est légitime de suspecter un risque d'insuffisance surrénalienne au cours des hypocholestérolémies chroniques, d'autant plus $s^{\prime}$ il s'agit d'une forme sévère avec des taux de cholestérolémie effondrés. Cependant, la littérature ne rapporte pas à notre connaissance de cas d'insuffisance surrénalienne aigue, et les tests de stimulation par ACTH ne révèlent qu'une légère baisse chez des sujets porteurs $\mathrm{d}^{\prime} \mathrm{AB}$ ou d'HB alors que la cortisolémie basale est normale (Illingworth et al., 1982 ; Illingworth et al., 1980). De même en réanimation, l'existence d'une hypocholestérolémie secondaire par exemple à un état infectieux sévère n'altère que légèrement la réponse initiale surrénalienne mais sans diminuer la sécrétion totale de cortisol (Arem et al., 1997).

En conclusion, I'hypocholestérolémie chronique ne suffit pas à induire un état d'insuffisance surrénalienne susceptible d'avoir une expression clinique.

\section{Autres conséquences}

La malabsorption intestinale des lipides présente dans les hypocholestérolémies familiales peut altérer la croissance chez l'enfant, et induire des complications neurologiques ou ophtalmiques par carence en vitamines $A$ et $E$ qui sont des vitamines liposolubles.

En effet, la carence en vitamine $E$ plasmatique est associée à une baisse de sa concentration dans les fibres nerveuses périphériques (Traber et al.,
1987) probablement en lien avec la dégénérescence neurologique axonale parfois observée. Si aucun traitement n'est débuté précocement, idéalement avant l'âge de deux ans, cette atteinte neurologique peut se manifester dès l'enfance initialement par une baisse des potentiels d'action sensoriels aux potentiels évoqués et à l'électromyogramme (Peretti et al., 2009), puis par une perte des réflexes achilléens; puis une diminution de la proprioception, une dysarthrie et une ataxie (Gauthier et Sniderman, 1983 ; Lacaille et al., 1989 ; Roy et al., 1987 ; Strich et al., 1993).

Enfin, dans I'AB et I'HB homozygote, une dégénérescence rétinienne pigmentaire décelable dès la petite enfance est responsable d'une diminution de l'acuité visuelle ou de la vision nocturne, elle évolue rarement jusqu'à la cécité (Muller et al., 1977). Soulignons, que dans la RCM les atteintes ophtalmiques restent modérées (micronystagmus, diminution de la vision nocturne ou des couleurs) (Peretti et al., 2009 ; Roy et al., 1987).

\section{Conclusion}

Les conséquences métaboliques de la malabsorption intestinale des lipides peuvent être soit bénéfiques comme une très probable diminution du risque cardiovasculaire, ou bien au contraire néfastes par les carences associées en vitamines liposolubles, acides gras essentiels ou augmentation du niveau de stress oxydant. Les complications métaboliques peuvent varier selon le type d'hypocholestérolémie en question : l'atteinte neurologique prédomine pour les formes sévères non traitées $d^{\prime} A B, d^{\prime} H B$ homozygote et à un degré moindre de RCM; alors qu'un risque accru de fibrose hépatique existe pour certaines formes asymptomatiques $\mathrm{d}^{\prime} \mathrm{HB}$ hétérozygotes.

La caractérisation des hypocholestérolémies familiales a permis de développer de nouveaux concepts thérapeutiques pour l'hypercholestérolémie comme les inhibiteurs de la MTP ou de I'ApoB. II persiste des hypocholestérolémies génétiques dont la cause n'est pas identifiée, permettant d'espérer le développement futur de nouvelles approches hypocholestérolémiantes une fois leur étiologie découverte. 
Remerciements. Nous remercions Monsieur Léonce Carré pour son aide dans la mise en forme du manuscrit.

\section{RÉFÉRENCES}

ANSES. Actualisation des apports nutritionnels conseillés pour les acides gras. Maisons-Alfort : ANSES, 2011.

Arem R, Ghusn H, Ellerhorst J, Comstock JP. Effect of decreased plasma low-density lipoprotein levels on adrenal and testicular function in man. Clin Biochem 1997; 30 : 419-24.

Avigan MI, Ishak KG, Gregg RE., Hoofnagle JH. Morphologic features of the liver in abetalipoproteinemia. Hepatology 1984 ; 4 : 1223-6.

Bosner MS, Lange LG., Stenson WF, Ostlund $\mathrm{RE}$, Jr. Percent cholesterol absorption in normal women and men quantified with dual stable isotopic tracers and negative ion mass spectrometry. J Lipid Res 1999 ; 40 : 302-8.

Chandler CE, Wilder DE, Pettini JL, et al. Cp346086: An mtp inhibitor that lowers plasma cholesterol and triglycerides in experimental animals and in humans. J Lipid Res 2003 ; 44 : 1887-901.

Cohen JC, Boerwinkle E, Mosley TH, Jr, Hobbs $\mathrm{HH}$. Sequence variations in pcsk9, low Idl, and protection against coronary heart disease. N Engl J Med 2006 ; 354 : 1264-72.

Cohen I, Pertsemlidis A, Kotowski IK, Graham R, Garcia CK, Hobbs HH. Low Idl cholesterol in individuals of african descent resulting from frequent nonsense mutations in pcsk9. Nat Genet $2005 ; 37$ : 161-5.

Crooke RM, Graham MJ, Lemonidis KM, Whipple CP, Koo S, Perera RJ. An apolipoprotein $\mathrm{b}$ antisense oligonucleotide lowers Idl cholesterol in hyperlipidemic mice without causing hepatic steatosis. I Lipid Res 2005 ; $46: 872-84$.

Elias N, Patterson BW, Schonfeld G. Decreased production rates of vldl triglycerides and apob-100 in subjects heterozygous for familial hypobetalipoproteinemia. Arterioscler Thromb Vasc Biol $1999 ; 19$ : 2714-21.

Ford ES, Li C, Zhao G, Mokdad AH. Concentrations of low-density lipoprotein cholesterol and total cholesterol among children and adolescents in the united states. Circulation 2009 ; 119 : 1108-15.

Fouchier SW, Sankatsing RR, Peter J, et al. High frequency of apob gene mutations causing familial hypobetalipoproteinaemia in patients of dutch and spanish descent. J Med Genet 2005 ; 42 : e23.

Gauthier S, Sniderman A. Action tremor as a manifestation of chylomicron retention disease. Ann Neurol 1983; 14 : 591.

Hercberg S, Preziosi P, Briancon S, et al. A primary prevention trial using nutritional doses of antioxidant vitamins and minerals in cardiovascular diseases and cancers in a general population: The su.Vi.Max studydesign, methods, and participant characteristics. Supplementation en vitamines et mineraux antioxydants. Control Clin Trials $1998 ; 19: 336-51$.

Illingworth DR, Kenny TA, Orwoll ES. Adrenal function in heterozygous and homozygous hypobetalipoproteinemia. J Clin Endocrinol Metab $1982 ; 54$ : 27-33.

Illingworth DR, Orwoll ES, Connor WE. Impaired cortisol secretion in abetalipoproteinemia. J Clin Endocrinol Metab 1980 ; 50 : 977-9.

Jones B, Jones EL, Bonney SA, et al. Mutations in a sar $1 \mathrm{gtpase}$ of copii vesicles are associated with lipid absorption disorders. Nat Genet $2003 ; 34: 29-31$.

Kim E, Campbell S, Schueller O, et al. A smallmolecule inhibitor of enterocytic microsomal triglyceride transfer protein, slx-4090: Biochemical, pharmacodynamic, pharmacokinetic, and safety profile. I Pharmacol Exp Ther 2011 ; 337 : 775-85.

Lacaille F, Bratos M, Bouma ME, Jos J, Schmitz J, Rey J. Anderson's disease. Clinical and morphologic study of 7 cases. Arch Fr Pediatr 1989 ; $46: 491-8$.

Lambert D, Daubrosse E, Steinmetz J, Siest G, Debry G. Reference values of plasma and lipoprotein cholesterol and triacylglycerols in healthy children. Arch Fr Pediatr 1984; 41 : 145-50.

Leppert M, Breslow JL, Wu L, et al. Inference of a molecular defect of apolipoprotein $b$ in hypobetalipoproteinemia by linkage analysis in a large kindred. J Clin Invest 1988; 82 : 847-51.

Muller DP, Lloyd JK, Bird AC. Long-term management of abetalipoproteinaemia. Possible role for vitamin e. Arch Dis Child 1977 ; 52 : 209-14.

$\mathrm{NIH}$. The lipid research clinics population studies data book. BETHESDA, National Heart and Lung Institute, 1980, 1.

Partin JS, Partin JC, Schubert WK, McAdams AJ. Liver ultrastructure in abetalipoproteine- mia: Evolution of micronodular cirrhosis. Gastroenterology 1974 ; 67 : 107-18.

Peretti N, Roy CC, Sassolas A, et al. Chylomicron retention disease: A long term study of two cohorts. Mol Genet Metab 2009 ; 97 : 136-42.

Peretti N, Sassolas A, Roy CC, et al. Guidelines for the diagnosis and management of chylomicron retention disease based on a review of the literature and the experience of two centers. Orphanet J Rare Dis $2010 ; 5: 24$.

Roy CC, Levy E, Green PH, et al. Malabsorption, hypocholesterolemia, and fat-filled enterocytes with increased intestinal apoprotein b. Chylomicron retention disease. Gastroenterology 1987 ; 92 : 390-9.

Sankatsing RR, Fouchier SW, de Haan S, et al. Hepatic and cardiovascular consequences of familial hypobetalipoproteinemia. Arterioscler Thromb Vasc Biol 2005 ; 25 : 1979-84.

Schonfeld G, Patterson BW, Yablonskiy DA, et al. Fatty liver in familial hypobetalipoproteinemia: Triglyceride assembly into vldl particles is affected by the extent of hepatic steatosis. J Lipid Res 2003 ; 44 : 470-8.

Sehayek E, Nath C, Heinemann T, et al. $U$-shape relationship between change in dietary cholesterol absorption and plasma lipoprotein responsiveness and evidence for extreme interindividual variation in dietary cholesterol absorption in humans. / Lipid Res 1998 ; 39 : 2415-22.

Silvain M, Bligny D, Aparicio $\mathrm{T}$, et al. Anderson's disease (chylomicron retention disease): A new mutation in the sara2 gene associated with muscular and cardiac abnormalities. Clin Genet 2008 ; 74 : 546-52.

Strich D, Goldstein R, Phillips A, et al. Anderson's disease: No linkage to the apo $b$ locus. J Pediatr Gastroenterol Nutr 1993 ; 16 : 257-64.

Tarugi P, Averna M. Hypobetalipoproteinemia: Genetics, biochemistry, and clinical spectrum. Adv Clin Chem 2011 ; 54 : 81-107.

Traber MG, Sokol RJ, Ringel SP, Neville HE, Thellman CA, Kayden HJ. Lack of tocopherol in peripheral nerves of vitamin e-deficient patients with peripheral neuropathy. $N$ Engl J Med 1987 ; 317 : 262-5.

Wetterau JR, Aggerbeck LP, Bouma ME, et al. Absence of microsomal triglyceride transfer protein in individuals with abetalipoproteinemia. Science 1992 ; 258 : 999-1001.

Wu J, Kim J, Li Q, et al. Known mutations of apob account for only a small minority of hypobetalipoproteinemia. / Lipid Res 1999 ; 40 : 955-9. 